\title{
Síndrome urémico hemolítico en pediatría
}

\author{
Hemolytic uremic syndrome in pediatrics
}

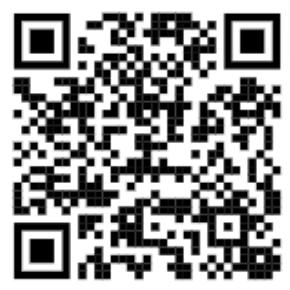

\author{
'Dra. Ishtar Estefanía Saborío Cervantes \\ Investigadora independiente, Heredia, Costa Rica \\ ishtar-8@hotmail.com \\ https://orcid.org/0000-0002-0106-9953 \\ ${ }^{2}$ Dra. María del Pilar Durán Monge \\ Investigadora independiente, San José, Costa Rica \\ mpdm07@hotmail.com \\ https://orcid.org/0000-0002-7562-9044 \\ ${ }^{3}$ Dr. David Villalobos Bonilla \\ Investigador independiente, Heredia, Costa Rica \\ davidvb77@gmail.com \\ https://orcid.org/0000-0002-5369-8663 \\ CORREGIDO \\ $15 / 03 / 2019$ \\ ACEPTADO \\ $27 / 03 / 2019$
}

RECIBIDO

$10 / 03 / 2019$
${ }^{1}$ Médico general, graduada de la Universidad de de Ciencias Médicas (UCIMED), investigadora independiente, Heredia, Costa Rica. Código médico: 15214

${ }^{2}$ Médico general, graduada de la Universidad de Costa Rica (UCR), investigadora independiente, San José, Costa Rica. Código médico: 15136.

${ }^{3}$ Médico general, graduado de la Universidad de Ciencias Médicas (UCIMED),

investigador independiente, Heredia, Costa Rica. Código médico: 15388

\section{RESUMEN}

El Síndrome Urémico Hemolítico constituye una microangiopatía trombótica, es una de las principales causas de lesión renal aguda en niños, además tiene un alto riesgo de morbimortalidad. Su etiología es variable, con factores infecciosos y/o genéticos e incluso puede llegar a ser secundario a una patología. Dentro de los hallazgos clínicos se presenta lesión renal aguda, trombocitopenia y anemia hemolítica microangiopática, más de la mitad de los pacientes presentan diarrea acuosa con disentería, acompañada de otros síntomas gastrointestinales como dolor abdominal, náuseas y vómitos. Otras manifestaciones que se presentan en la minoría de pacientes son a nivel cardiaco, neurológico, oftalmológico y dermatológico.

Para el diagnóstico se requiere primero identificar clínicamente el Síndrome Urémico Hemolítico y posterior a esto es necesario indagar sobre la causa del mismo. Los exámenes de laboratorio que se requieren para el diagnóstico incluyen hemograma, pruebas de función renal, pruebas de función hepática, examen general de orina y frotis de heces, además de exámenes complementarios para identificar su causa.

El tratamiento se debe instaurar lo antes posible con el fin de evitar o reducir las complicaciones agudas y las secuelas a largo plazo, siendo el tratamiento de soporte el manejo esencial.

PALABRAS CLAVE: Síndrome hemolítico-urémico, Escherichia coli, 
toxina Shiga, microangiopatías trombóticas, trombocitopenia, lesión renal aguda.

\begin{abstract}
The Hemolytic Uremic Syndrome constitutes a thrombotic microangiopathy, it is one of the main causes of acute kidney injury in children, it also has a high risk of morbimortality. Its etiology is variable, with infectious and / or genetic factors and may even be secondary to a pathology. The clinical findings include acute kidney injury, thrombocytopenia and microangiopathic hemolytic anemia, more than half of the patients have watery diarrhea with dysentery, accompanied by other gastrointestinal symptoms such as abdominal pain, nausea and vomiting. Other manifestations that occur in the minority of patients are cardiological, neurological, ophthalmological and dermatological. For the diagnosis, it is first necessary to identify the Hemolytic Uremic Syndrome clinically and after that it is necessary to investigate the cause of it. Laboratory tests that are required for diagnosis include a blood count, kidney function tests, liver function tests, a general urinalysis and stool smear, and complementary tests to identify the cause. Treatment should be established as soon as possible in order to avoid or reduce acute complications and long-term sequelae, with supportive treatment being the essential management.
\end{abstract}

KEYWORDS: Hemolytic-uremic syndrome, Escherichia coli, Shiga toxins, thrombotic microangiopathies, thrombocytopenia, acute kidney injury.

\title{
INTRODUCCIÓN
}

El Síndrome Urémico Hemolítico, denominado a partir de ahora como SUH, se describió por primera vez en 1950 en tres niños que presentaron diarrea con sangre, edema y convulsiones. En el año 1955 el pediatra Conrad Gasser y sus colaboradores reportaron cinco niños con manifestaciones clínicas similares que murieron de necrosis cortical, es a partir de este momento que se denomina al SUH como la triada de trombocitopenia, anemia hemolítica microangiopática y lesión renal aguda (LRA) (1).
El origen del SUH clásicamente se ha asociado a la bacteria Escherichia coli productora de la toxina Shiga (SUHSTEC) $y$ en menor medida por infecciones invasivas provocadas por la bacteria Streptococcus pneumoniae. Existen otras causas ocasionadas por alteraciones genéticas de la vía del complemento, además de condiciones adquiridas que pueden desencadenar un SUH, tales como fármacos, distintos agentes infecciosos, patologías autoinmunes, entre otros.

A pesar de la diferente etiología, las diversas formas de SUH tienen en común el estado protrombótico y 
proinflamatorio a nivel endotelial, que conlleva a la triada mencionada anteriormente (2).

Este síndrome tiene un alto riesgo de morbimortalidad, que puede provocar secuelas permanentes en el paciente, y es una causa importante de LRA, razón por la cual es de suma importancia la identificación temprana de la patología realizando el diagnóstico con los hallazgos clínicos característicos y apoyándose con datos de laboratorio, además del inicio rápido del tratamiento.

El presente artículo constituye una revisión bibliográfica que tiene como objetivo describir las principales características, clasificación, hallazgos clínicos, diagnóstico y tratamiento del Síndrome urémico hemolítico en Pediatría.

\section{DEFINICIÓN}

EI SUH es una de las principales causas de LRA en la población pediátrica, afectando primordialmente a niños en edad preescolar (3). Pertenece al grupo de microangiopatías trombóticas (MAT) con lesión endotelial inicial, principalmente con afectación renal debido a la presencia de un trombo formado por fibrina y plaquetas a nivel de los capilares y las arteriolas, además de inflamación de las células endoteliales y la separación de la membrana basal glomerular (4).

Se manifiesta clásicamente como la tríada de anemia hemolítica microangiopática, trombocitopenia y LRA, asimismo con su ocasional enfermedad diarreica sanguinolenta que está presente según su etiología $(5,6)$

\section{EPIDEMIOLOGÍA}

EI SUH típico se debe a una infección por E. coli enterohemorrágica productora de la toxina shiga siendo su serotipo el 0157:H7; es la causa más frecuente de SHU en pediatría representando el $90 \%$ de casos, afectando a las niñas con más frecuencia que los niños (6). La incidencia de SUH STEC es mayor en niños menores de 5 años siendo en Europa y América del Norte de 1.9 a 2.9 casos por 100.000 niños, se cree que es debido a que los anticuerpos anti-STEC se desarrollan en una edad más tardía. La presencia de SUH-STEC en América Latina es diez veces más alta que en otros continentes por ejemplo, el caso de Argentina de 10 a 17 casos por cada 100.000 habitantes menores de 5 años (4). El SUH por S. Pneumoniae (SUH $\mathrm{SP})$ representa el $5 \%$ y el SUH de otras causas (atípico) corresponde de 5\% a 10\% (4). En relación al SUH atípico, este se produce con igual frecuencia en niños y niñas. En un registro de SUH atípico de 516 pacientes en edad pediátrica, el 39\% de los pacientes desarrollaron la enfermedad antes de los 18 años de edad y aproximadamente el $44 \%$ de los niños experimentaron su primer episodio antes de los 2 años (7).

Existe mayor riesgo de desarrollar SUH en zonas ganaderas por la alta posibilidad de contaminación de agua y alimentos (8).

Un dato adicional es el período de incubación del STEC que corresponde a un tiempo de 1 a 8 días y la excreción de la bacteria por heces puede persistir durante más de 3 semanas después de la infección (8).

Finalmente, el SUH conforma la primera causa de LRA e hipertensión arterial en 
niños y constituye la segunda causa de insuficiencia renal crónica (1).

\section{CLASIFICACIÓN}

A partir de las investigaciones realizadas sobre este síndrome se han planteado diversas clasificaciones, una de ellas es SUH diarrea positivo (SUH D+), que es producido principalmente por STEC y SUH diarrea negativo (SUH D-), el cual abarca el resto de tipos de SUH, sin embargo, esta clasificación tiene ciertas limitaciones, ya que el SUH D+ puede presentarse sin diarrea, y el SUH Dpuede presentar este síntoma (3). Limitaciones como la recién nombrada han llevado a la aparición de la actual clasificación de SUH típico y atípico; el primero de estos se debe a bacterias productoras de la toxina Shiga y el término "atípico", se ha designado al SUH que no se asocia a infección por STEC ni a otra patología o condición coexistente $(1,9)$. Por este motivo, se han planteado otras formas de clasificación según el agente causal la cual se describe a continuación (4).

\section{Inducido por agentes infecciosos}
a. SUH STEC O también denominado SUH típico.

El SUH constituye la complicación más grave de las infecciones por STEC (10). Aproximadamente el $15 \%$ de los niños que presentan enterocolitis por $\mathrm{E}$. coli progresan a SUH, esto se ha asociado a una corta edad, y al género femenino que junto a la presencia de leucocitosis aumentan la posibilidad de desarrollar SUH.
La mayoría de las cepas de E. coli enterohemorrágica expresan una adhesina llamada intimina que permite a la subunidad A de la toxina Shiga ingresar al torrente sanguíneo y posteriormente unirse al endotelio por medio de la globotriaosilceramida (Gb3), que también se encuentra en los túbulos renales. Adicionalmente, ocurre una endocitosis de la toxina, esta causa inactivación ribosómica e inhibición de la síntesis proteica, que conlleva a la muerte celular.

Es necesario también mencionar que la toxina Shiga ejerce una actividad proinflamatoria y protrombótica a nivel glomerular, lo cual induce a la secreción del factor de von Willebrand y la activación del complemento $(1,2)$.
b. SUH asociado a Shigella dysenteriae tipo 1.

Esta bacteria al igual que la $\mathrm{E}$. coli es productora de la toxina Shiga, por lo que puede desencadenar un SUH típico (2).

\section{c. SUH asociado a Streptococcus pneumoniae.}

Ocurre posterior a una neumonía complicada por empiema o meningitis. Se ha demostrado la unión de proteínas que expresa la bacteria del neumococo al Factor $\mathrm{H}$, lo que inhibe la acción de esta (2). El S. pneumoniae produce neuroaminidasas que remueven el ácido $\mathrm{N}$-acetilneuramínico de la superficie de las células, de esta forma se expone el antígeno Thomsen-Friedenreich conocido como antígeno $\mathrm{T}$ que se encuentra en los eritrocitos, plaquetas y en el endotelio, la reacción de los anticuerpos contra este antígeno produce 
anemia hemolítica, trombocitopenia y lesión microvascular (1).

d. Otros agentes infecciosos: VIH e influenza H1N1 (11).

En el caso del VIH este provoca toxicidad en las células endoteliales, pero gracias a la incorporación de la terapia antirretroviral los casos han disminuido. Por su parte, la influenza H1N1, puede desencadenar un SUH en pacientes con mutación de la diacilglicerol quinasa $\varepsilon$ (4).

\section{SUH atípico}

La patogénesis del SUH atípicos surge de defectos genéticos o adquiridos en la regulación de la vía alterna del complemento (7).

a. Mutaciones en los genes del complemento:

Las mutaciones más comunes que se presentan son en el factor $\mathrm{H}$, factor I y en la proteína cofactor de membrana (MCP) (11).

Estas mutaciones conllevan a una activación no controlada de la vía alterna del complemento, que al mismo tiempo genera una sobreproducción del complejo de ataque a la membrana y se desencadena la muerte de células endoteliales y edema, lo que conduce a un estado protrombótico a nivel subendotelial, además se activa el sistema de coagulación. Por su parte, se da un aumento en la C5a, la cual es una anafilotoxina que produce quimiotaxis, activación leucocitaria y endotelial, genera también un aumento en la permeabilidad de los vasos, inflamación y trombosis, que culmina en una obstrucción a nivel de la microvasculatura (1).

b. Errores innatos del metabolismo de la cobalamina $\mathrm{C}$ :

La cobalamina $C$ es un cofactor para la metionina sintasa, que transforma la homocisteína a metionina, también es un cofactor de la metiltransferasa que participa en la conversión de metilmanolil Co $A$ a succinil CoA, por lo que un error en la función de la cobalamina $\mathrm{C}$ produce metilamonio academia con homocistinuria (6).

c. Mutaciones en la diacilglicerol quinasa $\varepsilon$ :

Esta proteína intracelular, se expresa en el endotelio, en plaquetas y en podocitos, se ha identificado en formas autosómicas recesivas de SUH atípico que ocurre en el primer año de vida (9).

La pérdida de función de esta confiere un estado protrombótico a nivel de la microvasculatura, ya que inactiva el diacilglicerol que contiene ácido araquidónico, que es un activador de la proteina kinasa $\mathrm{C}$ que promueve la trombosis, por lo tanto, cuando su función está alterada se genera un aumento en factores protrombóticos como el factor de von Willebrand y disminuyen factor de crecimiento vascular endotelial $(2,6,7)$.

\section{Coexistente con otras enfermedades o condiciones:}

Son casos que se presentan de forma esporádica, desencadenados por diferentes factores, algunos de los cuales se mencionan a continuación (4): 
- Drogas y medicamentos: inhibidores de la calcineurina como ciclosporina A y tacrólimus, drogas citotóxicas, quinina, anticonceptivos orales, inhibidores del factor de crecimiento vascular endotelial, quetiapina, quimioterapéuticos como gemcitabina y mitomicina, antiagregantes plaquetarios como el clopidogrel y la ticlopidina, drogas de abuso como la cocaína $(2,8)$.

- Embarazo: el SUH desencadenado por el embarazo ocurre principalmente en el periodo posparto (6).

- Enfermedades autoinmunes: lupus eritematoso sistémico, síndrome antifosfolípido, esclerodermia, vasculitis asociadas a ANCA, entre otras (12).

- Hipertensión maligna.

- Nefropatía preexistente.

- Neoplasias: cáncer de próstata, gástrico, cáncer pulmonar, cáncer de mama, linfoma, entre otros.

En donde pueden existir émbolos intravasculares a nivel tumoral, con activación de la coagulación y proliferación de la pared vascular (4).

- Trasplante de órgano sólido o de células madre: generalmente el SUH ocurre en los primeros meses posteriores al trasplante, esto también se relaciona con el uso de inhibidores de calcineurina en estos pacientes, ya que es en dicho periodo cuando se utilizan mayores dosis de dichos fármacos (6).

\section{HALLAZGOS CLÍNICOS}

Los signos comunes de las MAT incluyen anemia hemolítica microangiopática, con daño isquémico a algunos órganos, generalmente al riñón y el cerebro (12). EI SUH se presenta con distintos síntomas y signos, estos dependen del tiempo de evolución, severidad y órganos involucrados según el grado de afectación MAT (4). La mayoría de los pacientes presentan un inicio abrupto de la enfermedad (7).

Algunos hallazgos clínicos producidos por el grado de anemia incluyen palidez, fatiga, soplo sistólico y taquicardia, a pesar de la trombocitopenia el sangrado espontáneo y las petequias son poco frecuentes (13).

Existen síntomas gastrointestinales como enfermedad diarreica que inicia de 3 a 8 días posterior a la ingesta de alimentos contaminados, la misma se manifiesta de forma acuosa, siendo disentérica en el $70 \%$ de las ocasiones, asociada a dolor abdominal, náuseas y vómito en el $30 \%$ al $40 \%$ de los pacientes, en casos severos se presenta enterocolitis, pancreatitis, colitis hemorrágica, perforación íleo-colónica, prolapso rectal, colestasis y peritonitis $(1,2)$.

Otros de los hallazgos clínicos los constituyen las alteraciones renales que incluyen hematuria y proteinuria, disminución de la tasa de filtración glomerular e incremento en la creatinina (7). En el caso de la microalbuminuria, esta se considera un indicador temprano de afectación renal por hiperfiltración, algunos estudios demuestran 
microalbuminuria posterior al SUH en el $32 \%$ de los casos a los 3 años y el $22 \%$ a los 5 años. El 25\% de los niños puede presentar afectación renal a largo plazo como proteinuria, hipertensión arterial e insuficiencia renal crónica (10).

También es posible la aparición de una serie de diversas manifestaciones neurológicas como visión borrosa, cefalea, letargia, convulsiones, irritabilidad, un evento cerebrovascular isquémico o hemorrágico, coma, hemiparesia, edema cerebral y ceguera cortical $(1,12)$. El compromiso neurológico ocurre debido a la disfunción endotelial, la hipertensión arterial y las alteraciones hidroelectrolíticas, especialmente la hiponatremia (14). Las complicaciones neurológicas se asocian a un aumento en la mortalidad (15).

Además, se encuentran las manifestaciones cardiacas como la isquemia miocárdica secundaria al aumento de volumen manejado y a la hipertensión donde se ha reportado acumulación sanguínea en el espacio pericárdico, produciendo finalmente un taponamiento cardiaco $(8,16)$

Asimismo, existen otras manifestaciones como diabetes mellitus y pancreatitis (8).

En el SUH típico presenta ocasionalmente disentería y un antecedente de infección urinaria por su etiología más común de $\mathrm{E}$. coli. El SUHSP ocurre en pacientes con sepsis grave por este organismo asociado a neumonía o en un $30 \%$ con meningitis (4).

En contraste con el SUH típico, en el SUH atípico el comienzo de la enfermedad puede estar relacionado con diferentes eventos intermitentes desencadenantes como gastroenteritis virales, influenza, vacunación, estrés, etc (4). Cabe mencionar que el $42 \%$ de los pacientes con SUH atípico se asocia a diarrea o gastroenteritis e infección de vías respiratorias superiores (7).

Finalmente, las manifestaciones extra renales reportadas en el $25 \%$ de los pacientes con SUH atípico son gangrena a nivel de falanges media y distal, que incluso puede llevar a amputación, estenosis de las arterias craneales y extra craneales y a nivel ocular se puede presentar isquemia retiniana $y$ hemorragia del vítreo $(12,13)$.

\section{DIAGNÓSTICO}

Los estudios a realizar inicialmente se deben enfocar en el diagnóstico de la MAT y posteriormente en determinar la causa del SUH, para esto es necesario descartar las causas infecciosas, genéticas y estudiar posibles patologías coexistentes 0 condiciones como embarazo y uso de medicamentos que pueden provocar la patología (12).

El diagnóstico del SUH se basa clínicamente con la presencia de la triada clásica de anemia hemolítica microangiopática, trombocitopenia y LRA. Esto se sustenta con exámenes de laboratorio como hemograma completo, frotis sanguíneo, estudios de función renal y examen de orina (6).

La anemia hemolítica microangiopática se determina con un valor de hemoglobina menor a $8 \mathrm{~g} / \mathrm{dL}$, test de coombs negativo (excepto en el $\mathrm{SUH}$ ocasionado por S. pneumoniae), un frotis sanguíneo que presenta esquistocitos, descenso de haptoglobulina, hiperbilirrubinemia indirecta, reticulocitosis e incremento de Lactato Deshidrogenasa (mayor a 460 U/L) $(1,6)$. 
La trombocitopenia depende de la gravedad de la enfermedad (se encuentra ausente en el $15 \%$ al $20 \%$ ), se define con niveles menores de 150.000 plaquetas; comúnmente se presenta con cifras menores de 40.000 plaquetas usualmente no se detecta clínicamente sangrados ni púrpura como consecuencia de esta cantidad $(1,4)$.

La lesión renal aguda se puede manifestar de diversas formas según su severidad, como proteinuria, hematuria o hasta falla renal, se debe analizar los niveles de creatinina, nitrógeno ureico (BUN), diuresis y cifras hipertensivas (6). Para confirmar el diagnóstico de SUH STEC se debe detectar la bacteria E. coli 0157:H7 en heces, PCR o títulos de anticuerpos en suero (5). Adicionalmente, para el diagnóstico de SUH atípico es útil la medición de C3, $\mathrm{C} 4$, factor $\mathrm{H}$, factor $\mathrm{I}$, mutaciones genéticas e investigación de enfermedad autoinmune (8). Cerca del $60-70 \%$ de los casos de SUH atípico presenta una mutación en los genes del complemento 0 anticuerpos contra el factor $\mathrm{H}$ (9).

Aunque la biopsia no es necesaria para el diagnóstico definitivo su histopatología se presenta de tres maneras: MAT glomerular, MAT arterial y necrosis cortical parcheada (1).

\section{DIAGNÓSTICO DIFERENCIAL}

Existen muchas condiciones que comparten características clínicas con el $\mathrm{SUH}$, entre ellas se encuentran varias afecciones que pueden presentarse con hallazgos concomitantes de anemia, trombocitopenia y lesión renal aguda. Algunas de ellas se presentan a continuación:
- La coagulación intravascular diseminada (CID): se diferencia de la SUH por la presencia de pruebas de coagulación anormales, tales como el tiempo de tromboplastina parcial activada y protrombina prolongado y los niveles elevados de productos de degradación de fibrina y dímero $D$. En la mayoría de los casos, la CID ocurre en pacientes pediátricos que se encuentran en un estado de salud grave, tales como pacientes en shock séptico o que han sufrido una lesión tisular masiva (6).

- La púrpura trombocitopénica trombótica (PTT): constituye una de las 2 principales causas de MAT (TABLA 1.). La PTT es la causa más común de MAT en adultos, y se debe al desarrollo de autoanticuerpos contra la proteasa que separa el factor de von Willebrand $y / o$ a la actividad deficiente de la proteasa de escisión del factor de von Willebrand causada por mutaciones del gen ADAMTS13. El dato característico de la PTT es la deficiencia grave de la proteasa ADAMTS13 que corta el factor de von Willebrand. La PTT pediátrica es poco frecuente, habitualmente se caracteriza por una participación neurológica mayor que la renal, los niños afectados suelen presentarse al nacer con anemia hemolítica y trombocitopenia. La participación renal a menudo ocurre más tarde en la vida y tiene un curso progresivo. La PTT se distingue del SUH por una actividad ADAMTS13 anormalmente baja $(6,13)$.

- Vasculitis sistémica: los pacientes con vasculitis normalmente no tienen una enfermedad diarreica prodrómica 
y presentan otros síntomas sistémicos como artralgias y erupción cutánea. Además, la afectación neurológica característica en pacientes con vasculitis es periférica en lugar de central (6).

\begin{tabular}{|c|c|}
\hline $\begin{array}{l}\text { Síndrome } \\
\text { urémico } \\
\text { hemolítico }\end{array}$ & $\begin{array}{l}\text { Toxina Shiga (bacterias } \\
\text { productoras de la toxina) } \\
\text { - Escherichia coli } \\
\text { - Shigella dysenteriae } \\
\text { Streptococcus pneumoniae } \\
\text { Defecto en el metabolismo de la } \\
\text { cobalamina } \\
\text { SUH atípico } \\
\text { Desórdenes en la regulación del } \\
\text { complemento } \\
\text { Desórdenes en la coagulación }\end{array}$ \\
\hline $\begin{array}{l}\text { Púrpura } \\
\text { trombocitopenica } \\
\text { trombotica } \\
\text { (deficiencia de } \\
\text { ADAMTS13) }\end{array}$ & $\begin{array}{l}\text { Adquirida (autoanticuerpos) } \\
\text { Congénita (herencia recesiva) }\end{array}$ \\
\hline Secundario & $\begin{array}{l}\text { HIV } \\
\text { Embarazo } \\
\text { Síndrome antifosfolipídico } \\
\text { Malignidad } \\
\text { Medicación (quimioterapia, } \\
\text { inhibidores de la calcineurina) } \\
\text { Radioterapia } \\
\text { Trasplantes } \\
\text { Hipertensión severa }\end{array}$ \\
\hline
\end{tabular}

Fuente: Greenbaum L. Atypical Hemolytic Uremic Syndrome. Advances in Pediatrics 2014. (13)

- Lupus eritematoso sistémico: se muestra especialmente en adolescentes y mujeres jóvenes, esta se asemeja al SUH por la presencia de anemia y en los pacientes con nefritis lúpica por su trombocitopenia y LRA (13).

- Dengue hemorrágico: manifiesta trombocitopenia, LRA y anemia al igual que SHU sin embargo, la serología para dengue y los tiempos de coagulación alterados lo diferencia del SUH (1).

\section{TRATAMIENTO}

El manejo oportuno del SUH es lo más importante en estos casos, además de un traslado rápido a un centro especializado para proporcionar el tratamiento indicado (8). El tratamiento de soporte sigue siendo el enfoque principal en este síndrome, se basa en la corrección hídrica y electrolítica adecuada según la necesidad del paciente, este manejo ha mejorado el pronóstico renal, mermado la necesidad de diálisis y además disminuyendo las secuelas a largo plazo.

Se puede presentar tanto aumento como disminución del volumen intravascular, de acuerdo con esto se procede con el manejo de volumen. La disminución en el volumen intravascular puede ocurrir a causa de diarrea, vómitos o por baja ingesta oral, en este caso se debe retornar a un estado de euvolemia. El aumento en el volumen intravascular se debe manejar con restricción hídrica, incluso puede ser necesario realizar diálisis, especialmente cuando existe un daño cardiaco o pulmonar (17).

Es importante valorar e individualizar la corrección de la hipertensión, se sugiere utilizar medicamentos bloqueadores de los canales de calcio como nifedipina $0.25 \mathrm{mg} / \mathrm{kg}$ o amlodipina $0.1 \mathrm{mg} / \mathrm{kg}$ (1).

En la causa neumocóccica es necesario el manejo antibiótico de la infección subyacente (amoxacilina o cefalosporina de tercera generación en caso de meningitis), caso contrario al SUH-STEC que es controversial el uso de antibiótico $(4,8)$. 
Las indicaciones para iniciar terapia de reemplazo renal son: uremia sintomática, BUN > $100 \mathrm{mg} / \mathrm{dl}$, sobrecarga severa de líquidos (mayor a $15-20 \%$ del peso corporal), no respuesta a diuréticos, desórdenes electrolíticos y ácido base refractarios e incapacidad de suplir la nutrición o hidratación por la necesidad de restricción hídrica $(1,2)$.

Se indica transfusión de glóbulos rojos empacados en caso de anemia severa (menor a $8 \mathrm{~g} / \mathrm{dl}$ con repercusión hemodinámica), en los pacientes con hiperkalemia o sobrecarga hídrica esta se debe realizar durante diálisis $(1,17)$. La transfusión de plaquetas es controversial debido al riesgo de empeorar el cuadro de MAT, su uso es restringido en pacientes con un sangrado significativo (6).

En el SUH atípico y en el SUH STEC con complicaciones graves neurológicas se aconseja el uso temprano del anticuerpo monoclonal anti C5 Eculizumab, siendo este el primer y único anticuerpo aprobado para esta patología $(18,19)$. Además, es de suma importancia al utilizar el medicamento Eculizumab la vacunación contra meningococo, neumococo y Haemophilus influenzae tipo $B$, además de profilaxis anti meningococo $(1,8)$.

El uso del plasma fresco congelado sigue siendo controversial, aun así, en los casos de SUH atípico en los que no se pueda iniciar con Eculizumab se utiliza la transfusión de plasma fresco congelado, este por su contenido de complemento, remueve los factores mutados y los anticuerpos, el síndrome ocasionado por factor $\mathrm{H}$ responde mejor a este tratamiento $(1,8,9)$. No se recomienda el uso de plasma en SUH SP (8).
Los inmunosupresores como ciclofosfamida, corticoides y rituximab están indicados en el SUH atípico ocasionado por factor $H(1,4)$.

Finalmente, el trasplante hepático es una posibilidad en el SUH atípico mediado por complemento, ya que el hígado es el precursor de estas proteínas (19). Además, el trasplante renal se realiza valorando el grado de cronicidad renal, siendo útil en estos casos, es necesario realizar estudio genético previamente buscando mutaciones que puedan hacer recurrir el SUH (1).

\section{CONCLUSIÓN}

EI SUH se considera la principal causa de LRA en la población pediátrica, llegando a ser un gran riesgo para la vida del paciente dado las diversas afecciones sistémicas que están involucradas. Por ello la importancia para el clínico de conocer lo mejor que pueda dicha patología, además de ejecutar un diagnóstico temprano en busca de mejorar la supervivencia renal y de esta manera evitar la progresión de la enfermedad que conlleva a la expresión de múltiples manifestaciones clínicas del tipo neurológicas, renales, endocrinas, cardiovasculares entre otras. EI diagnóstico se basa en signos clínicos que orientan a isquemia orgánica (principalmente riñón y cerebro) y datos de laboratorio que confirmen su triada característica: anemia hemolítica microangiopática, trombocitopenia y lesión renal aguda. La amplia variedad de posibles causas del SUH y de los datos característicos en el laboratorio, hace que su diagnóstico acertado constituya un verdadero reto clínico, 
además es de suma importancia diferenciarlo de otros diagnósticos con los cuales comparte características clínicas y hallazgos de laboratorio. El inicio temprano del tratamiento mejorará el pronóstico renal y sus demás complicaciones.

\section{REFERENCIAS}

1. Bello D. Síndrome hemolítico urémico. CCAP [Internet]. 2016 [Consultado 4 marzo 2019]; 14(4):57-68. Disponible en: https://scp.com.co/wp-content/uploads/2016/02/14-4-5.pdf

2. Cody E, Dixon B. Hemolytic Uremic Syndrome. Pediatr Clin N Am [Internet]. 2019 [Consultado 3 marzo 2019]; 66(1):235-246. https://doi.org/10.1016/j.pcl.2018.09.011

3. Jenssen, G., Vold, L., Hovland, E., Bangstad, H-J., Nygård, K., Bjerre, A. Clinical features, therapeutic interventions and long-term aspects of hemolytic-uremic syndrome in Norwegian children: a nationwide retrospective study from 1999-2008. BMC Infectious Diseases [Internet]. 2016 [Consultado 1 marzo 2019]; 16:285. https://doi.org/10.1186/s12879-016-1627-7

4. Fakhouri F, Zuber J, Frémeaux-Bacchi V, Loirat C. Haemolytic uraemic síndrome. Seminar. 2017 Aug 12 ; 390(10095):681-696. https://doi.org/10.1016/S0140-6736(17)30062-4.

5. Lunn A, Forbes T. Haematuria and proteinuria in childhood. Paediatrics and Child Health. 2019 [Consultado 9 marzo 2019];28(8):315-321. https://doi.org/10.1016/i.paed.2012.02.011

6. Niaudet, P., Gillion, O. Overview of hemolytic uremic syndrome in children. UpToDate [Internet]. 2019 [Consultado 7 marzo 2019]. Disponible en: https://www.uptodate.com/contents/complement-mediatedhemolytic-uremic-syndrome

7. Dixon B, Gruppo R. Atypical Hemolytic Uremic Syndrome. Pediatr Clin N Am [Internet]. 2018 [Consultado 7 marzo 2019]; 65(3):509-525. Disponible en: https://doi.org./10.1016/j.pcl.2018..02.003

8. Kaur A., Kerecuk L. Haemolytic uraemic syndrome. Paediatrics and Child Health. 2016; 26(8): 344-348. https://doi.org/10.1016/j.paed.2012.01.002

9. Loirat $\mathrm{C}$, Fakhouri F, Ariceta G, et al. An international consensus approach to the managementof atypical hemolytic uremic syndrome in children. Pediatr Nephrol [Internet]. 2016 [Consultado 6 marzo 2019]; 31(1):15-39. https://doi.org./10.1007/s00467-015-3076-8

10. Cubillos $M$, Salas $P$, Zambrano $P$. Microalbuminuria en pacientes pediátricos con diagnóstico de síndrome hemolítico urémico. Revista Chilena de Pediatría [Internet]. 2015 [Consultado 6 marzo 2019]; 86(2):92-96. http://dx.doi.org/10.1016/i.rchipe.2015.04.019

11. Brocklebank V, Johnson S, Sheerin TP, Marks SD, Gilbert RD, Tyerman K, et al. Factor H autoantibody is associated with atypical hemolytic uremic syndrome in children in the United Kingdom and Ireland. Kidney $\begin{array}{llllll}\text { Int } \quad \text { [Internet]. } 2017 \quad \text { [Consultado } & 5 & \text { marzo }\end{array}$ https://doi.org/10.1016/i.kint.2017.04.028

12. Cavero T, Alonso M. Where are we with haemolytic uremic syndrome?. Med Clin (Barc) [Internet]. 2018 [Consultado el 10 de marzo 2019]; 151(8):329-335. http://dx.doi.org/10.1016/j.medcle.2018.08.010

13. Greenbaum L. Atypical Hemolytic Uremic Syndrome. Advances in Pediatrics [Internet]. 2014 [Consultado 8 marzo 2019;61(1):335-356. http://dx.doi.org/10.1016/j.yapd.2014.04.001 
14. López M, Huete I, Hernández M. Compromiso cerebrovascular agudo en síndrome hemolítico urémico: descripción de dos casos pediátricos. Rev Chil pediatría [Internet]. 2017 [Consultado 8 marzo 2019]; 88(5):640-646. http://dx.doi.org/10.4067/S0370-41062017000500011

15. Spartà G, Nef S, Neuhaus TJ, Latal B, Buder K, Laube GF. Neurodevelopmental long-term outcome in children after hemolytic uremic syndrome. Pediatr Nephrol [Internet]. 2015 [Consultado 5 marzo 2019]; 30(3):503-513. https://doi.org./10.1007/s00467-014-2950-0

16. Rigamonti D, Simonetti GD. Direct cardiac involvement in childhood hemolytic-uremic syndrome: case report and review of the literature. Eur J Pediatr [Internet]. 2016 [Consultado 8 marzo 2019]; 175(12):1927-1931. https://doi.org/10.1007/s00431-016-2790-y

17. Niaudet P, Mattoo TK, Kim MS. Treatment and prognosis of Shiga toxin-producing Escherichia coli (STEC) hemolytic uremic syndrome (HUS) in children. Uptodate [Internet]. 2019 [Consultado 7 marzo 2019]. Disponible en: https://www.uptodate.com/contents/treatment-and-prognosis-of-shiga-toxinproducing-escherichia-coli-stec-hemolytic-uremic-syndrome-hus-in-children

18. Greenbaum L, Fila M, Ardissino G, Al-Akash S, Evans J, Henning P, et.al. Eculizumab is a safe and effective treatment inpediatric patients with atypical hemolytic uremic syndrome. Kidney International [Internet]. 2016 [Consultado 11 marzo 2019]; 89(3):701-711. http://dx.doi.org/10.1016/i.kint.2015.11.026

19. Niaudet P, Gillion O. Complement-mediated hemolytic uremic syndrome. Uptodate [Internet]. 2019 [Consultado 8 marzo 2019]. Disponible en: https://www.uptodate.com/contents/complement-mediatedhemolytic-uremic-syndrome 